\title{
Effet perçu d'une communauté d'apprentissage sur la motivation des enseignants du secondaire dans leur développement professionnel
}

Aziz Rasmy

Maroc

Thierry Karsenti

Université de Montréal

Canada
Perceived impact of a learning community on the motivation of high school teachers

in their professional development

doi:10.18162/fp. 2017.352

\section{$R$ ésumé}

Le présent article a pour objectif de décrire et de comprendre les effets d'une communauté d'apprentissage

(CA) sur la motivation des enseignants' dans leur développement professionnel lié à l'intégration des

TIC. La collecte de données a été effectuée à l'aide d'entrevues individuelles auprès du personnel enseignant du secondaire. Les résultats mettent en évidence toute

la pertinence d'une communauté d'apprentissage par l'autonomie rendue aux enseignants, la valorisation qu'on accorde à leurs pratiques et les possibilités de prendre en compte le profil particulier de même que les besoins individuels de ses membres plutôt que de tenir compte des faiblesses des personnes.

\section{Mots-clés}

Développement professionnel, communauté d'apprentissage, motivation autodéterminée, besoins psychologiques fondamentaux, intégration des TIC.

Abstract

This article aims to describe and understand the effects of a learning community (AC) on the motivation of teachers in their professional development in the implementation of IT in the classroom. The acquisition of the data has been done using individual interviews of middle school and early high school teaching staff. The results show the pertinence of a learning community, from the autonomy granted to teachers, to the valorisation given to their work and from the possibility to take into account the particularities and the individual needs of its members, rather than using the deficiencies of its constituents.

\section{Keywords}

Professional development, learning community, selfdetermined motivation, basic psychological needs, integration of ICT.

\section{Introduction}

La notion d'éducation tout au long de la vie s'est progressivement imposée dans plusieurs régions du globe, dont le Québec (Ministère de l'Éducation, du Loisir et du Sport du Québec [MELS], 2008). Cet intérêt est de plus en plus justifié dans le monde de l'éducation par le fait que l'adaptation des enseignants aux changements est devenue une exigence fondamentale dans un contexte où l'évolution des savoirs progresse à une rapidité sans cesse croissante (OCDE, 2005). À cet effet, il est important pour les enseignants en exercice de s'inscrire dans une perspective d'apprentissage continu actif où ils développent leurs compétences professionnelles en vue d'une plus grande réussite scolaire chez les élèves (Berry, Daughtrey et Wieder, 2010; Cave et Mulloy, 2010; Fullan, 2011).

Dans cette vision, les conceptions de la formation continue actuelle s'inscrivent de plus en plus dans une démarche collective intégrant les savoirs théoriques et pratiques (Charlier, 1998; Donnay et Charlier, 2008). Cette intégration vise le développement des compétences spécifiques et la maîtrise de l'intervention pédagogique et non simplement l'acquisition des connaissances contribuant peu au changement de pratiques dans la classe.

Selon le ministère de l'Éducation, du Loisir et du Sport (MELS), « la formation continue des enseignants est confrontée à certaines tensions au sein du corps enseignant, voire à une certaine résistance au changement » $(2008$, p. 1$)$. On peut penser particulièrement à la résistance inhérente à l'intégration des technologies de l'information et de la communication. En effet, selon le $\mathrm{MEQ}^{2}$ (2001), l'intégration des TIC à l'école sollicite des compétences particulières de la part du personnel enseignant. Dans cette perspective, Karsenti et Larose (2005) mentionnent que l'un des enjeux décisifs de cette intégration est certes leur appropriation par les enseignants. 
Depuis quelques années, on réfère souvent au mode de fonctionnement comme communauté d'apprentissage (CA) pour favoriser le développement professionnel des enseignants et la réussite scolaire des élèves (Leclerc, Philon, Dumouchel, Laflamme et Giasson, 2013). Cette démarche repose sur la collaboration, le partage d'expériences et la responsabilisation des participants dans leur apprentissage professionnel. Soulignons à ce propos que les systèmes scolaires de plusieurs provinces canadiennes ont adopté récemment le mode de fonctionnement de CA (Leclerc et Labelle, 2013).

Certains chercheurs et praticiens font remarquer que les structures de formation axées sur la communauté d'apprentissage suscitent l'intérêt et l'engagement individuel et collectif des enseignants dans leur développement professionnel (Charlier, 1998; Dionne et Couture, 2013; Moreau, Leclerc et Stanké, 2013). Par contre, les facteurs permettant d'expliquer l'engagement des enseignants dans leur développement professionnel sont peu connus. À ce propos, Schieb et Karabenick (2011) soutiennent qu'une grande partie de la recherche porte sur la motivation dans l'apprentissage des élèves, mais peu sur la motivation des enseignants. Cette perspective liée à la motivation des enseignants dans le développement professionnel nous interpelle dans la présente étude.

Ainsi, la question principale de cette étude est la suivante : la mise en œuvre d'une communauté d'apprentissage (CA) influence-t-elle la motivation des enseignants dans leur développement professionnel? L'enjeu principal de cette recherche est donc de décrire et d'expliquer en quoi la mise en œuvre d'une CA peut favoriser l'accroissement de la motivation des enseignants dans leur développement professionnel. Il est donc intéressant de s'arrêter sur l'impact des activités de formation sur la motivation à s'engager dans le développement professionnel du personnel enseignant. Dans les sections qui suivent, nous présentons le cadre théorique à la base de cette recherche. Ensuite seront présentés la méthodologie, les résultats et une discussion en guise de conclusion.

\section{Cadre conceptuel}

Le présent cadre théorique vise à préciser les éléments à la base du questionnement de cette recherche. Dans un premier temps, nous présentons un aperçu sur la communauté d'apprentissage. Ensuite, nous traitons les déterminants de la motivation selon la théorie de l'autodétermination.

\section{Le concept de communauté d'apprentissage}

Depuis le début des années 1990, l'approche de communauté d'apprentissage (CA) est de plus en plus partie prenante de la littérature (Hord et Sommers, 2008, cités dans April et Bouchamma, 2014). Il ressort de la littérature que les activités de développement professionnel efficace et durable doivent créer des structures de soutien continu et développer des communautés de pratique favorisant la collaboration et le partage des pratiques professionnelles (Peters et Savoie-Zajc, 2013). Pour sa part, Charlier (1998) avance que les enseignants sont plus disponibles à s'impliquer dans les activités de formation où existe un climat de collégialité et de communauté de pratique. Selon cette chercheuse, les discussions, les échanges, la collaboration et le soutien mutuel sont des facteurs contribuant au développement des sentiments de confiance et d'appartenance sociale. 
La communauté d'apprentissage repose sur la théorie de la pratique sociale développée par Wenger (2005). Selon cette théorie, le processus de construction d'une pratique professionnelle dans un milieu de travail ne se limite pas uniquement à des facteurs individuels : il est un processus influencé aussi par la collaboration et les interactions des praticiens partageant des valeurs et des intérêts relatifs à leur pratique professionnelle. Ce mode de développement professionnel est vécu comme un processus éminemment social pendant lequel les praticiens s'inscrivent dans une dynamique de partage et d'échanges ainsi que dans une posture réflexive (Wenger, 2005). À partir de cette vision sociale de l'apprentissage professionnel, Wenger (2006) décrit une communauté d'apprentissage comme « une démarche par laquelle un groupe de personnes qui partagent une préoccupation ou une passion pour quelque chose quelles font et apprennent afin d'améliorer leurs pratiques en interagissant de façon régulière » (p. 1 , traduction libre).

Dans la présente recherche, nous avons retenu la définition synthèse de la communauté d'apprentissage suivante : « la CAP, désignée comme outil d'accompagnement du personnel enseignant, permet à la fois un engagement individuel et collectif de formation continue, des échanges d'idées entre collègues quant à la pertinence des pratiques didactiques et pédagogiques et une analyse réflexive sur sa propre pratique » (Hord et Sommers, 2008, cités dans April et Bouchamma, 2014, p. 1).

En somme, la communauté d'apprentissage est vue comme mode de développement professionnel, mené par de petits groupes de pairs qui s'engagent pour prendre en main leur apprentissage et la construction de leur savoir-enseigner. Savoie-Zajc insiste notamment sur la " présence d'un projet d'apprentissage conjoint, d'une intention de développement ainsi que d'une volonté de croissance commune » (SavoieZajc, 2013, citée dans Leclerc et Labelle, 2013, p. 3). Cette forme de développement professionnel fournit aux enseignants, à la direction d'école et aux divers intervenants des occasions pour échanger et se soutenir. En conséquence, le vécu et l'expérience de chacun des participants sont mis à contribution pour développer une compréhension et une analyse des réalités afin d'amener plus d'élèves à réussir. Ainsi, lorsque ces activités d'apprentissage sont partagées de manière formelle par les membres d'une équipe, les changements sont plus porteurs de succès. La section qui suit sera consacrée à analyser les déterminants de la motivation qui se trouvent au cœur de ce projet de recherche.

\section{La motivation autodéterminée}

Dans la présente recherche, nous adoptons la perspective de la motivation autodéterminée de Deci et Ryan (2000) pour étudier les facteurs motivationnels dans l'apprentissage professionnel des enseignants. En effet, la théorie de l'autodétermination (TAD) est actuellement à la base de plusieurs recherches permettant de comprendre le phénomène de la motivation ainsi que ses liens avec l'engagement professionnel (Assor, Kaplan, Feinberg et Tal, 2009). La TAD est une théorie de motivation générale issue de la distinction entre la motivation intrinsèque et extrinsèque qui démontre que plus le contrôle est interne sur le continuum d'autodétermination, plus la motivation est intrinsèque et la performance est élevée. À l'opposé, plus le contrôle des comportements est externe, plus la motivation est extrinsèque.

Selon Deci et Ryan (2000), l'être humain possède une tendance innée à s'actualiser. Cette tendance intégrative repose sur l'environnement dans lequel l'individu évolue et sur le soutien lié à la satisfaction

de ses besoins psychologiques fondamentaux comme les sentiments d'autonomie, de compétence et 
d'appartenance sociale. Ces différentes formes de motivation permettent d'expliquer le comportement humain dans différents contextes tels que le travail, l'apprentissage scolaire, la formation, etc. Dans les lignes qui suivent, nous présenterons trois dimensions relatives aux activités susceptibles de soutenir la motivation dans un contexte de formation professionnelle des enseignants.

\section{Soutien à l'autonomie}

Le besoin d'autonomie renvoie à une volonté de se percevoir à l'origine de ses comportements (Deci et Ryan, 2000). La notion même de l'autonomie se trouve ancrée dans une vision de la personne, vue comme un être humain auteur de ses actes et des actions professionnelles qu'il engage (Fullan, 2011). En effet, lorsqu'un individu satisfait son besoin d'autonomie dans une activité, il éprouve généralement une motivation autodéterminée pour celle-ci et perçoit que ses comportements découlent de ses choix et de sa volonté (Deci et Ryan, 2000). Il valorise pleinement ses actions et en retire de la satisfaction et du plaisir. L'individu assume alors pleinement son engagement dans le comportement qui est désiré.

Un comportement autonome implique que l'apprenant perçoit qu'il possède un contrôle sur son apprentissage. Par exemple, le sentiment de contrôle d'un enseignant lors d'une démarche de développement professionnel renvoie à la possibilité du sujet de choisir les activités de formation et à la façon dont il contrôle et dirige son propre apprentissage. Somme toute, un climat qui soutient l'autonomie peut être identifié comme celui marqué par un style motivationnel encourageant le choix et la participation à la prise de décision.

\section{Soutien au besoin de compétence}

Le besoin de compétence se définit comme le désir de vouloir être efficace dans ses relations avec l'environnement. Ce sentiment explique l'existence de forces orientant les comportements de l'individu vers des activités favorisant la reconnaissance, la confiance et le succès. Les effets recherchés sont en rapport avec l'accomplissement qui pousse le sujet à démontrer son habileté au regard de luimême ou des autres, à relever des défis et à améliorer ses capacités. Ainsi, lorsqu'un individu satisfait son besoin de compétence dans une activité, il se sent généralement compétent envers celle-ci. Selon Fullan (2011), ce sentiment pourrait engendrer un sentiment d'accomplissement du devoir moral et devient une force permettant d'assurer les changements de pratiques de façon continue. Le besoin de compétence est soutenu par la reconnaissance, les rétroactions positives et par les expériences de succès dans des activités de développement professionnel (Dionne et Savoie-Zajc, 2011).

\section{Soutien à l'appartenance sociale}

Le besoin d'appartenance sociale d'un individu correspond au besoin de se sentir accepté et soutenu dans ses relations interpersonnelles et d'appartenir à une communauté ou à un groupe social (Deci et Ryan, 2000). Selon Deci et Ryan (2000), la motivation autodéterminée serait plus en mesure d'apparaître dans un contexte de collaboration, d'échange et de partage. Dans le contexte de la formation professionnelle, on réfère souvent à la collaboration, à la qualité des relations interpersonnelles et au sentiment d'appartenance du participant aux autres membres du groupe (Dionne et Savoie-Zajc, 2011). 
La collaboration est souvent perçue comme un moyen pour favoriser le développement professionnel des enseignants, notamment par le biais de communautés d'apprentissage afin d'aider les enseignants à améliorer leur pratique (Dionne et Savoie-Zajc, 2011). Ainsi, les échanges de pratiques et les prises de conscience collective peuvent engendrer une culture commune, un langage partagé par une communauté et des sentiments d'appartenance à un groupe (Donnay et Charlier,2008). Dans le même sens, les résultats de l'étude de Rhéaume et al.(1999) montrent que les enseignants en exercice accordent une importance à la formation continue dans la mesure où ces activités pourraient être une occasion de rencontrer des enseignants ayant des préoccupations semblables aux leurs. Ils ont mentionné des situations d'apprentissage professionnel associées notamment à un climat propice à la collaboration, à un sentiment d'appartenance au groupe et à la rupture de l'isolement.

En référence à ces dimensions, nous avons établi, à partir des travaux de Keller (1987), les activités d'apprentissage susceptibles de favoriser la motivation des apprenants. Le tableau 1 présente ces trois dimensions et les catégories correspondantes.

\section{Tableau 1}

Les dimensions et activités pédagogiques associées à la motivation en contexte de formation professionnelle.

Dimensions

\begin{tabular}{|c|c|}
\hline Latitude et responsabilité & 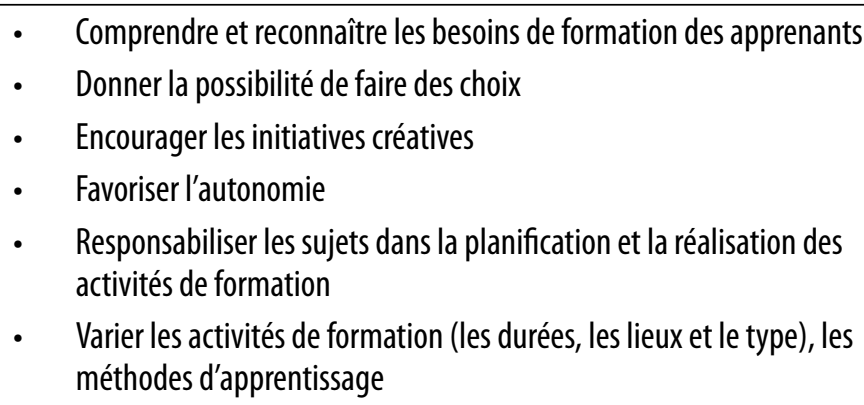 \\
\hline $\begin{array}{l}\text { Besoin de compétence et } \\
\text { d'accomplissement }\end{array}$ & $\begin{array}{l}\text { - } \text { Favoriser la confiance en soi } \\
\text { - } \quad \text { Proposer des tâches en lien avec les compétences des enseignants } \\
\text { - Donner une rétroaction positive } \\
\text { - } \quad \text { Aider les enseignants à fixer des buts réalisables pour faire vivre des } \\
\text { succès individuels et de groupe }\end{array}$ \\
\hline $\begin{array}{l}\text { Appartenance sociale et } \\
\text { collaboration }\end{array}$ & $\begin{array}{l}\text { - Mettre en place des méthodes qui favorisent la collaboration et la } \\
\text { coopération } \\
\text { - Établir une communauté d'apprentissage en dehors des ateliers de } \\
\text { formation } \\
\text { - Instaurer un climat agréable (humour, etc.) }\end{array}$ \\
\hline
\end{tabular}


En définitive, la motivation occupe une part importante dans les conceptions actuelles de l'apprentissage professionnel des enseignants. Elle est au cœur des dispositifs de développement professionnel, particulièrement dans le contexte d'adoption d'innovations pédagogiques comme l'intégration des TIC. Cependant, c'est à la base des facteurs motivationnels contextuels que les enseignants trouvent les ressources nécessaires pour déclencher ce processus d'appropriation de ces savoirs et ainsi s'engager continuellement dans leur développement professionnel. La recension d'écrits a permis de ressortir trois sources principales de la motivation, soit les dimensions suivantes : 1) la satisfaction du besoin de latitude et de la responsabilité; 2) la satisfaction du besoin de compétence et d'accomplissement et 3) la satisfaction du besoin d'appartenance sociale et de collaboration.

\section{Méthodologie}

Puisque la présente recherche consiste à approfondir la compréhension d'un phénomène assez peu documenté, la méthode de recherche qualitative interprétative nous semble particulièrement indiquée. Cette recherche repose prioritairement sur le point de vue nécessairement subjectif des participants afin d'arriver à mieux saisir la dynamique motivationnelle dans le développement professionnel (Spear, 1988). Ainsi, la collecte de données a été effectuée à l'aide d'entrevues individuelles semi-dirigées.

\section{Description du milieu de collecte des données}

Dans le but d'amener plus de jeunes à réussir, la direction du centre de formation des adultes à Montréal a mis en place, depuis l'année scolaire 2011-2012, une CA. Cinq enseignants de ce centre de formation participent volontairement à cette $\mathrm{CA}$. En collaboration avec la direction et l'équipe-école, la conseillère en orientation de ce centre de formation a guidé les enseignants dans cette démarche de développement professionnel qui visait à mieux soutenir les élèves $(n=34)$ à risque de difficultés scolaires dans leur réussite éducative.

Ce dispositif de développement professionnel se fonde sur une pédagogie centrée sur l'apprenant en valorisant la collaboration et la démarche d'enseignement en équipe (team teaching) permettant d'impliquer plusieurs enseignants représentant diverses matières. La CA adopte une structure organisationnelle souple favorisant l'implication des participants dans la gestion de leur formation et tient en compte leurs besoins et intérêts professionnels. Les thèmes des activités de développement professionnel se regroupent autour de quatre grands objectifs : 1) les pratiques liées à l'intégration des technologies de l'information et de la communication; 2) l'enseignement différencié; 3) le projet personnel et l'intégration socioprofessionnelle; 4) la motivation dans l'apprentissage.

Les membres du groupe se réunissent à la fin de chaque semaine sur une base formelle et régulière et également sur une base informelle au cours de l'année scolaire pour discuter des difficultés rencontrées relatives à la réussite des apprenants à risque, pour partager des expériences, pour expliciter leurs pratiques et pour planifier leurs besoins en formation. De façon générale, les stratégies utilisées par l'animatrice (la conseillère en orientation) dans les rencontres de groupe consistent à favoriser le sentiment de valorisation du travail enseignant, l'esprit d'équipe et le codéveloppement professionnel. Les résultats de cette CA sont très éloquents et montrent une nette amélioration du rendement scolaire et une diminution du décrochage scolaire des apprenants (Roy, Moussaid, Gnofame et Renaud, 2013) 3 . 
Des enseignantes et des enseignants, qui étaient déjà engagés dans une démarche de $\mathrm{CA}$, ont accepté de participer à des entrevues afin de permettre une meilleure compréhension de l'impact de cette démarche sur leur développement professionnel. En d'autres termes, leur participation aux entrevues permettrait la réalisation d'une analyse approfondie en vue de comprendre de quelle façon une démarche de CA pourrait leur apporter la motivation à poursuivre leur développement professionnel.

\section{Participants}

Afin de s'assurer que les données recueillies reflètent bien le point de vue du personnel impliqué dans la $\mathrm{CA}$, il est important de recueillir de l'information auprès du plus grand nombre possible de sujets. Ainsi, tous les enseignants $(n=5)$ engagés dans la mise en œuvre de la CA sont retenus pour participer aux entrevues individuelles semi-dirigées. Le fait de mener des entrevues avec tous les enseignants permet de donner un aperçu plus approfondi sur la dynamique motivationnelle que peut susciter ce dispositif pédagogique au sein du groupe. Les enseignants ont accepté volontairement de participer à notre collecte de données. La moyenne d'âge des enseignants interviewés est de 45,6 ans et la moyenne d'années d'expérience en enseignement est de 14,2 ans. Le tableau 2 présente des renseignements sur chacun des cinq participants aux entrevues individuelles.

Tableau 2

Profil des participants aux entreoues individuelles.

\begin{tabular}{cllll} 
Participants & Qualification & $\begin{array}{l}\text { Années } \\
\text { d'expérience }\end{array}$ & Âge & $\begin{array}{l}\text { Matières } \\
\text { enseignées }\end{array}$ \\
\hline Enseignante (1) & $\begin{array}{l}\text { Brevet en enseignement } \\
\text { secondaire et maîtrise dans le } \\
\text { domaine des sciences sociales }\end{array}$ & 13 ans & 46 & Français \\
\hline Enseignante (2) & $\begin{array}{l}\text { Brevet en enseignement } \\
\text { secondaire et maîtrise en } \\
\text { éducation }\end{array}$ & 16 ans & 44 & $\begin{array}{l}\text { Mathématiques et } \\
\text { sciences }\end{array}$ \\
\hline Enseignante (3) & Baccalauréat en français & 12 ans & 56 & Français \\
\hline Enseignant (4) & Baccalauréat en anglais & 11 ans & 36 & Anglais \\
\hline Enseignante (5) & $\begin{array}{l}\text { Brevet en enseignement } \\
\text { secondaire }\end{array}$ & 19 ans & 46 & Informatique \\
\hline
\end{tabular}

\section{Instruments de collecte de données}

Pour atteindre notre objectif qui a trait à la compréhension d'un phénomène social où l'intention n'est pas la généralisation, la collecte de données a été effectuée à l'aide d'entrevues individuelles semi-dirigées (Tashakkori et Teddlie, 2003). Comme le souligne Poupart (1997), l'entrevue est « l'un des meilleurs moyens pour saisir le sens que les acteurs donnent à leurs conduites, la façon dont ils se représentent le monde et la façon dont ils vivent leur situation » (p. 175). Nous avons opté pour l'entrevue semi-dirigée 
pour amener les participants à fournir des réponses liées à notre objectif, contrairement à l'entrevue non dirigée où le chercheur se contente d'enregistrer l'expérience de l'interviewé sans aucune orientation.

Au cours des entretiens, il était demandé aux participants d'exprimer leur point de vue sur des questions qui gravitent autour des éléments favorisant la motivation en formation invoqués dans notre cadre conceptuel. Le schéma d'entrevue (voir annexe) comporte une dizaine de questions qui ont pour but d'approfondir le thème étudié et de permettre à l'interviewé d'exprimer son point de vue sans pour autant laisser place à une discussion libre. Voici des exemples de questions posées : quelles sont les expériences qui ont influencé votre envie de participer à la CA? En quoi cela a-t-il eu un impact sur votre développement professionnel en matière d'intégration des TIC? Y a-t-il eu des expériences partagées qui ont été intéressantes pour votre implication? Si oui, pouvez-vous en donner un exemple?

\section{Démarche d'analyse de données}

L'analyse des données recueillies à l'aide d'entrevues individuelles s'est effectuée par la méthode d'analyse de contenu manifeste (Van der Maren, 1996). Pour ce faire, nous avons utilisé le logiciel d'analyse qualitative QDA Miner. La grille de notre codage est établie à partir des facteurs susceptibles de favoriser la motivation des enseignants dans la formation professionnelle relevée dans la recension des écrits et qui s'appuient sur les principales dimensions de la motivation autodéterminée de Deci et Ryan (2000). Par exemple, le code "possibilité de choisir les activités de formation » a trait au sentiment d'autonomie; le code "reconnaissance des efforts " concerne le besoin de compétence, le code « climat propice à la collaboration et à la formation » est lié au sentiment d'appartenance sociale. En définitive, l'analyse de contenu s'est effectuée comme elle est présentée dans le tableau 3, selon les principales étapes élaborées par Van der Maren (1996), à savoir la collecte des données pour la préanalyse, le codage des données et le traitement des données.

\section{Tableau 3}

Modèle général des étapes de l'analyse de contenu (adaptation du modèle de Van der Maren (1996).

\begin{tabular}{ll}
\hline Étapes & Caractéristiques \\
\hline 1 & Préanalyse des données recueillies (organisation des données) \\
\hline 2 & Définition des codes de classification des données recueillies \\
\hline 3 & Processus de catégorisation des données recueillies \\
\hline 5 & Codification des données \\
\hline 6 & Description scientifique des cas étudiés \\
\hline
\end{tabular}

À noter que nous avons adopté un processus de catégorisation et de classification mixte. Au cours du codage, sept codes ont été ajoutés et trois codes ont été supprimés. Au total, 212 unités de sens ont été dégagées des entrevues et une base de données informatisée a été constituée pour les analyser. 


\section{Résultats}

Cette section présente les résultats relatifs aux effets perçus de la CA sur la motivation dans le développement professionnel des participants. Il s'agit principalement des commentaires des participants liés au sentiment d'autonomie, de compétence et d'appartenance sociale.

Dimension liée au sentiment d'autonomie

L'analyse du discours des participants a fait ressortir trois aspects en lien avec le sentiment d'autonomie, soit la possibilité de choisir les thématiques, le sentiment d'être responsable de sa démarche de développement professionnel et l'autoformation.

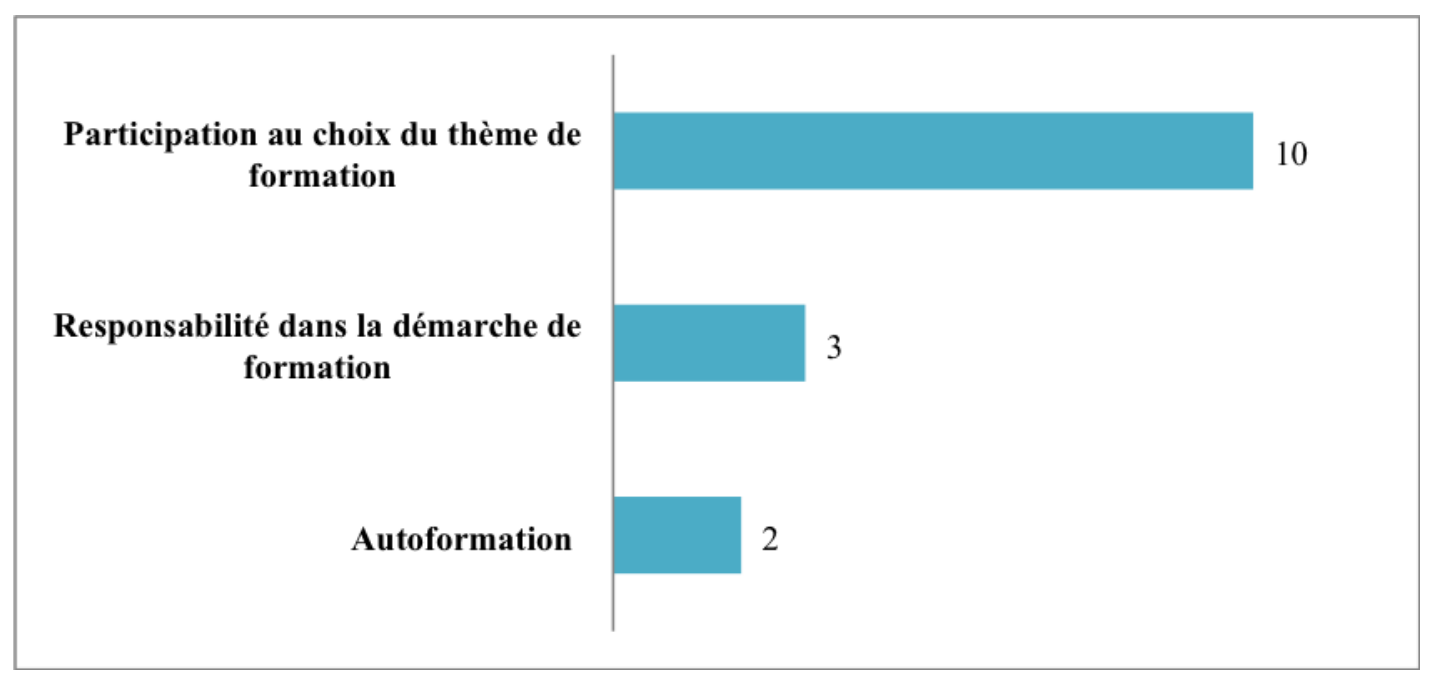

Figure 1

Fréquence des codes relatifs au sentiment d'autonomie.

\section{La possibilité de participer au choix des activités}

Il se dégage du discours des participants (10 commentaires) un intérêt pour les activités de formation qui laissent à l'enseignant la possibilité de choisir les thèmes de formation, les contenus, les intervenants, les horaires, etc. Par exemple, une participante rapporte : "je me sentais vraiment interpelée dans le choix des thèmes de formation, c'était vraiment tout le groupe qui faisait des suggestions ». Dans la perspective de faire des choix, une participante fournit un éclairage sur son intérêt à négocier a priori les activités de formation pour pouvoir les refuser si elles ne correspondaient pas à ce qui était souhaité.

\section{Sentiment de responsabilité}

On note également dans le discours des répondants des commentaires concernant la responsabilité des enseignants dans la gestion de leur démarche de développement professionnel (3 commentaires). Par exemple, une participante qui a pris part à la CA a évoqué les rôles et les responsabilités assumés 
par les enseignants dans les différents comités et les activités de développement professionnel dans le cadre de ce projet : "je suis toujours là pour participer aux rencontres, aux différentes activités; ça me permet de faire le point, me remettre en question, d'assumer mes responsabilités envers soi-même, la responsable du projet ». Elle ajoute que "le partage des responsabilités avait permis aux enseignants de s'approprier le projet, d'être plus engagés dans leur développement professionnel; chaque membre de l'équipe a mis la main à la pâte, a contribué à la réussite du projet ».

\section{L'autoformation}

Les pratiques d'autoformation ressortent également des témoignages des répondants ( 2 commentaires) comme la consultation des sites Internet, la lecture des livres, etc. Un participant rapporte : « moi, je suis une personne qui aime expérimenter et découvrir des nouvelles choses. Internet me permet de me former moimême, de trouver beaucoup de choses intéressantes, des nouvelles références pour me rendre plus efficace ». Dans le même sens, il affirme : " on a appris sur le tas, ce n'était pas une formation formelle, alors c'est vraiment sur le tas; on m'a expliqué, on l'essaie. Si ça ne marche pas, je retourne, c'est vraiment à ce niveau-là que cétait intéressant». Selon les commentaires des personnes, cette forme de développement professionnel leur permet d'être autonomes et de ressentir une satisfaction professionnelle.

\section{Dimension liée au besoin de compétence}

La deuxième partie des résultats porte sur les commentaires des participants relatifs au besoin de compétence. Comme illustré dans la figure 2, trois aspects concernant cette dimension ressortent : le sentiment de confiance, l'accomplissement et la reconnaissance.

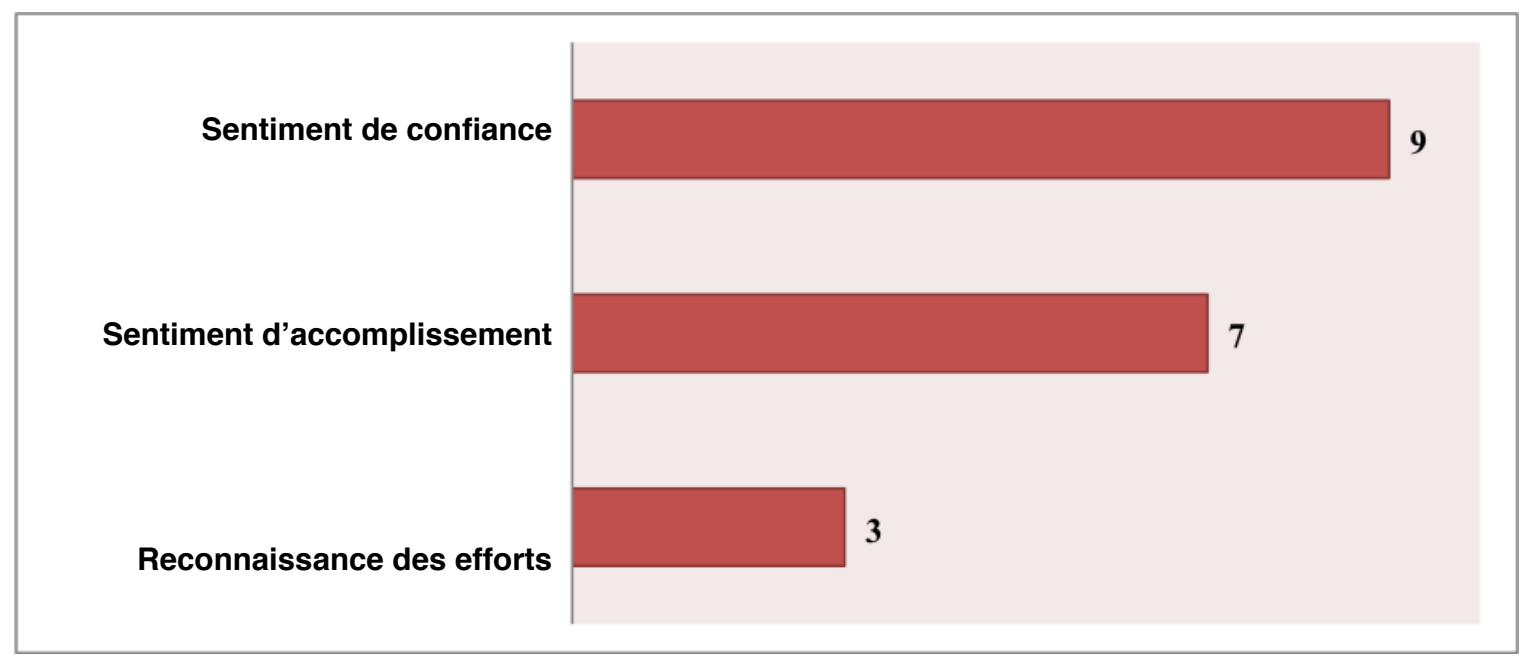

Figure 2

Fréquence des codes relatifs au besoin de compétence. 


\section{Sentiment de confiance}

Les participants ont évoqué des éléments ayant trait à la confiance $(9$ commentaires) dans le développement professionnel en intégration des technologies. Par exemple, une participante mentionne que la formation continue lui permet de se sentir beaucoup mieux outillée pour favoriser la réussite scolaire de ses élèves. Elle mentionne qu'elle participe à la formation continue en intégration des TIC d'abord pour se sentir plus compétente. Une autre participante signale aussi la dynamique des collègues vivant des situations comparables : «dans notre travail, on se débrouille quotidiennement tout seul; lorsquion rencontre les collègues puis quion constate que nos collègues sont confrontés aux mêmes difficultés et problèmes, puis quion échange et propose des choses pour trouver des solutions, c'est un élément clé de confiance selon moi [...] alors, c'est vraiment tout comme ça puis, en fait, ça a eu un impact pour que je puisse m’impliquer parce que je veux connaître, je veux me sentir compétente aussi, parce que ça a vraiment tout cet environnement-là qui a donné tout cela». Dans un autre ordre d'idées, une participante témoigne du soutien apporté par l'animatrice du projet de développement professionnel (communauté de pratique): "nous discutons beaucoup entre nous, la responsable du projet nous incite à dire notre opinion et à participer; elle nous fait confiance, nous encourage dans les moments difficiles».

\section{Sentiment d'accomplissement}

Le sentiment d'accomplissement ressort aussi du vécu des enseignants interrogés (7 commentaires). Par exemple, une participante apprécie la formation : "j'aime la formation continue et je n'admets pas le fait de ne plus mettre à jour mes connaissances pédagogiques et m’épanouir ». Elle mentionne : "j'avais un plaisir aussi à faire ça, à lire plusieurs textes partagés par mes collègues, à voir ce que je peux en retirer, à mener une recherche; j'aime ça». Un participant exprime que la formation continue permettait "de continuer d'apprendre, de progresser et d'enrichir ma culture tant au niveau personnel que professionnel ».

Dans la même perspective, une autre participante explique que la participation à des activités de formation la valorise beaucoup : "l'année prochaine, nous aurons un site de notre classe où on va mettre nos projets, où on peut intégrer les cours; les élèves pourront mettre des vidéos. On veut le faire, on veut l'intégrer, on va créer le site où il y aura une page pour commenter l'avis des élèves; le directeur est très ouvert ».

\section{Sentiment de reconnaissance}

Des propos en lien avec le sentiment de reconnaissance ressortent aussi du discours des enseignants (3 commentaires). Une participante a mentionné que les rétroactions de la coordinatrice du projet du développement professionnel et l'appui de la direction de l'école ont été une source de motivation à s'engager dans la formation continue. Le rôle joué par le conseiller pédagogique en intégration des TIC scolaires dans leur développement professionnel est mentionné aussi par les participants : «le conseiller m'informe souvent des pratiques nouvelles, des affaires qui pourront m'aider dans mon enseignement ». Pour une autre participante, le soutien et l'appui du conseiller pédagogique a été une grande source de motivation à son implication dans les activités de formation. 


\section{Dimension liée au sentiment d'appartenance sociale}

La troisième partie des résultats porte sur les commentaires des participants liés à l'appartenance sociale. Comme illustré dans la figure 3 , trois aspects rattachés à cette dimension ressortent : un climat propice à la collaboration, un sentiment d'appartenance au groupe et le sentiment de briser l'isolement.

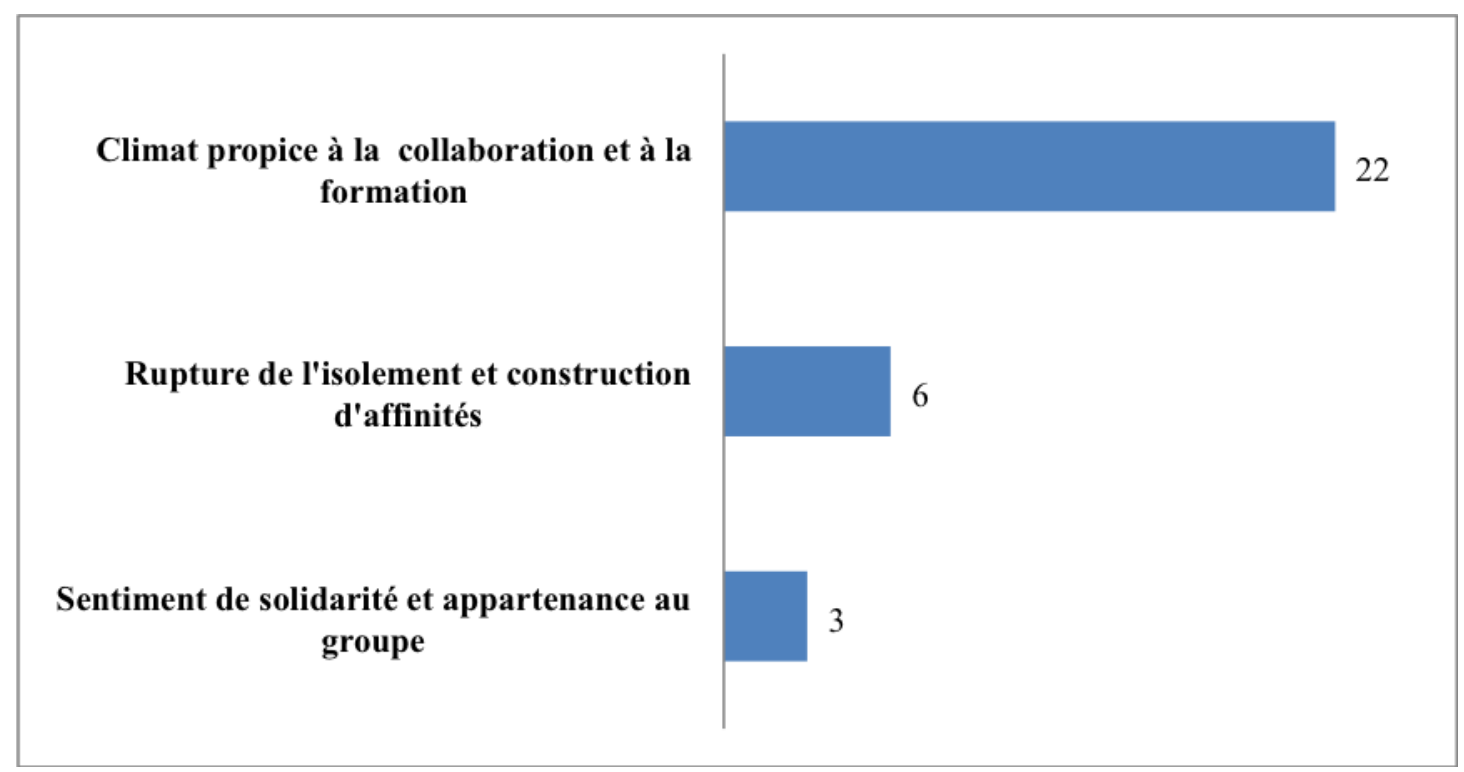

Figure 3

Fréquence des codes relatifs au sentiment d'appartenance sociale.

\section{Un climat propice à la collaboration et à la coopération}

L'analyse du discours des participants a permis de ressortir plusieurs propos en lien avec la collaboration et la coopération (22 commentaires). Par exemple, un participant rapporte : "j'ai appris beaucoup de mes collègues; on s'entraide beaucoup pour trouver les bonnes façons de changer le comportement des apprenants et les motiver dans leur apprentissage ». Une autre participante observe que le fait d'échanger avec des collègues qui enseignent aux mêmes classes d'élèves visant des buts et objectifs communs engendre une plus grande motivation à travailler en groupe. Elle mentionne aussi que «les rencontres sont riches, les personnes me soutiennent dans mon travail. C'est vraiment superbe, impeccable [...] j'ai toujours aimé avoir des gens avec qui je peux échanger des lectures, des auteurs, ça fait travailler».

Elle ajoute : «en fait, les activités de développement professionnel, c'est venu un peu avec le développement du groupe parce que je n'avais pas ces connaissances-là au départ. On travaille avec un conseiller pédagogique en informatique qui nous a poussés beaucoup, qui a pu nous donner toutes sortes de perspectives auxquelles on peut acquérir des connaissances en informatique... là, on se développe, on apprend sur le tas, on essaie des choses, et tout ça. Alors, c'est vraiment tout ça, puis en fait, ça a eu un impact pour que je puisse m’impliquer parce que je veux connaître, je veux me sentir compétente aussi, parce que c'est vraiment tout cet environnement-là qui a donné tout cela». 


\section{Rupture de l'isolement et construction d'affinités}

Un autre aspect qui ressort des propos des participants est l'impression de briser l'isolement et de bâtir des affinités. Ainsi, un participant mentionne que la formation est une occasion de partager les expériences professionnelles avec les pairs : "les échanges me permettent de mouvrir sur les autres, de créer des liens et de continuer à échanger en dehors de la formation ».

Dans la même perspective, une autre participante précise que son implication dans les activités de formation continue lui permet de "ne pas se sentir seule, d'atténuer le stress au travail et de briser l'isolement». Au fur et à mesure des échanges, elle a, selon elle, développé plus d'affinités avec ses collègues.

\section{Un sentiment de solidarité et d'appartenance au groupe}

Un autre aspect qui semble pousser les enseignants à s'engager dans leur développement professionnel est celui de l'entraide et de la solidarité. Une participante mentionne qu'elle a énormément apprécié le contexte dans lequel se déroule son développement professionnel : "cétait superbe et intéressant la relation entre tout le groupe; on travaille toujours en équipe, avec des collègues qui sentraident». Ainsi, un climat propice à la collaboration et à la coopération dépend de la dynamique qui sétablit dans le groupe en formation. Dans cette perspective, une autre participante ajoute : « ce que je trouve intéressant dans ce genre de formation, c'est que nous sommes très proches et très solidaires. C'est une force indéniable; de même, nous avons des relations beaucoup plus proches avec la coordinatrice du projet et la direction». Une participante souligne aussi l'entraide de manière informelle : " on s'entraide beaucoup, même en dehors des rencontres».

\section{Discussion}

Cette section présente la discussion des résultats relatifs à notre objectif de recherche qui était de déterminer les effets perçus du CA sur la motivation dans le développement professionnel.

Comme nous l'avons mentionné précédemment, la motivation dans le développement professionnel des enseignants est influencée par la nature et la qualité des interventions de formation continue offertes aux participants. Selon la théorie de l'autodétermination de Deci et Ryan (2000), la satisfaction des besoins psychologiques fondamentaux est une source de motivation dans la formation et dans l'apprentissage. Nous avons également vu, dans notre cadre conceptuel, que les déterminants de la motivation dans la formation continue se composent de plusieurs dimensions, dont les plus importantes examinées dans la présente étude sont la satisfaction des besoins psychologiques inhérents aux sentiments d'autonomie, de compétence et d'appartenance sociale. La satisfaction de ces besoins pourrait varier en fonction des tâches à réaliser et des conditions dans lesquelles celles-ci doivent être effectuées.

Pour la dimension d'autonomie, la théorie de l'autodétermination postule que la latitude et la responsabilité dont disposel'individu pour réaliser une activitéinfluencent la motivation autodéterminée. Les résultats de cette étude indiquent que le soutien à l'autonomie dans le cadre de la CA joue un rôle important dans le processus motivationnel des enseignants dans leur développement professionnel. 
Ainsi, un contexte de développement professionnel qui permet aux participants d'exprimer leurs attentes et leurs opinions, qui offre la possibilité de faire des choix et d'assumer des responsabilités, qui soutient l'autonomie facilite l'engagement des enseignants dans leur développement professionnel. Ces constats corroborent les résultats de recherche de Dionne et Couture (2010) qui indiquent que « les enseignants apprécient le contexte de choix et de liberté qu'offre la CA pour parler de ce qu'ils désirent et non pas se faire imposer des contenus » (p. 159). À cet égard, Fontaine, Savoie-Zajc et Cadieux (2013) soutiennent que l'enseignant est un professionnel autonome, engagé dans le perfectionnement continu de ses compétences et capable d'identifier ses besoins en vue de soutenir la réussite éducative de ses élèves.

Nous avons aussi vu se profiler d'autres aspects liés au besoin de compétence, soit la confiance, la reconnaissance et l'accomplissement. Il ressort des résultats de la présente recherche que le besoin de compétence est également important dans le soutien à la motivation du personnel enseignant. Ces résultats indiquent que la satisfaction du besoin de compétence se trouvant dans un contexte d'apprentissage qui tient compte de leurs besoins d'accomplissement, de fierté personnelle, de jugement positif, de valorisation et de reconnaissance. Ainsi, cette étude corrobore les résultats soulevés par Dionne et Savoie-Zajc (2011) qui notent que la présentation des réalisations et l'apport personnel dans une équipe sont, pour les enseignants en formation, des éléments contribuant à leur satisfaction personnelle et à l'actualisation de soi.

Nous avons aussi vu, auparavant, que le soutien au besoin d'appartenance sociale d'un individu a un effet positif sur la motivation dans l'apprentissage et la formation (Deci et Ryan, 2000). Le besoin d'appartenance sociale d'un individu renvoie à la nécessité de se sentir accepté et soutenu dans ses relations interpersonnelles et d'appartenir à une communauté ou à un groupe social (Deci et Ryan, 2000). Les résultats de notre recherche indiquent que les enseignants impliqués dans une démarche de CA valorisent les activités de formation collaboratives et les possibilités d'échanger avec leurs collègues plus expérimentés. Ces résultats rejoignent également les constats de Fontaine et al. (2013) concernant les conditions favorisant la collaboration dans le développement professionnel: « il doit y avoir existence d'un projet commun et certaines valeurs sont partagées [...] des relations signifiantes entre les personnes, et un sentiment de confiance, d'appartenance et de sécurité doit prévaloir » (p. 16).

Ainsi, à travers les échanges et la collaboration, les enseignants peuvent retirer des bénéfices sur les plans personnel et social. Par exemple, le fait d'échanger sur leurs pratiques, leurs valeurs et leurs croyances peut entraîner des retombées positives en matière de soutien affectif et d'appartenance au groupe (Savoie-Zajc, 2010). Dans le même sens, selon cette chercheuse, les facteurs relationnels comme les échanges socioaffectifs semblent des préalables aux échanges intellectuels (Savoie-Zajc, 2010). Ainsi, la mise en place de situations de collaboration et de coopération dans une CA apparaît cruciale pour la création d'interactions significatives et satisfaisantes entre les enseignants en formation. 


\section{Conclusion}

Les résultats de cette étude indiquent que le fonctionnement en CA présente un contexte pédagogique prometteur dans sa relation avec la motivation des enseignants dans leur développement professionnel. En effet, cette démarche qui repose sur l'autonomie du personnel, la liberté de choisir les activités de formation et d'être responsable de son propre développement professionnel contribue au développement d'un sentiment d'accomplissement et de satisfaction des enseignants en formation. C'est par la stimulation de l'autonomie individuelle, tout en travaillant en groupe, que les enseignants, en tant qu'adultes et ayant plusieurs années d'expérience professionnelle, peuvent s'impliquer dans les activités de formation qui leur sont proposées (Fullan, 2011).

Le climat dans lequel se déroule la $\mathrm{CA}$, caractérisée par la collaboration, les échanges et le partage des expériences professionnelles représente aussi une source de développement d'un sentiment d'appartenance sociale, de plaisir et de motivation intrinsèque. Cette démarche de développement professionnel offrant des contenus de formation qui répondent aux besoins des participants est susceptible d'influencer positivement l'engagement professionnel des enseignants. En somme, les résultats de la présente recherche tendent à appuyer les conclusions d'études (Cave et Mulloy, 2010; Leclerc et Labelle, 2013; Peters et Savoie-Zajc, 2013) mentionnant l'impact positif des activités du CA sur l'engagement professionnel des enseignants.

Tout bien considéré, au terme de cette étude il faut aussi noter quelques limites qui viennent nuancer les résultats présentés ici. D'abord, cette étude est associée à un nombre réduit de participants, ce qui empêche évidemment la généralisation des résultats. Une prochaine étude pourrait s'attaquer à cette limite en cherchant à collecter des données auprès d'un échantillon exhaustif. Ensuite, il serait pertinent d'effectuer des observations lors de la mise en ouvre de la CA. Cette méthode de collecte de données additionnelle permet de mieux associer les commentaires des participants et leur motivation dans leur développement professionnel. Aussi, à propos de la collecte de données, il aurait été éclairant d'interviewer les administrateurs de la CA pour mieux percevoir la façon dont ils se situent quant à la dynamique motivationnelle et au soutien qu'ils pourront apporter à ce sujet. Il serait aussi intéressant de mener une recherche dans un intervalle de temps plus large afin de mieux comprendre les effets réels d'une communauté d'apprentissage sur le soutien à la motivation dans le développement professionnel. Enfin, on ne peut pas nier que plus de recherches sont nécessaires afin de documenter les impacts de ce dispositif de formation sur la réussite scolaire des élèves.

\section{Références}

April, D. et Bouchamma, Y. (2014). Accompagnement du personnel enseignant par les communautés d'apprentissage professionnelles (CAP) : pratiques-clefs et caractéristiques des directions d'école. Formation et profession, 23(2), 1-14. http://dx.doi.org/10.18162/fp.2015.271

Assor, A., Kaplan, H., Feinberg, O. et Tal, K. (2009). Combining vision with voice: A learning and implementation structure promoting teachers' internalization of practices based on self-determination theory. Theory and Research in Education, 7(2), 234-243. http://dx.doi.org/10.1177/1477878509104328

Berry, B., Daughtrey, A. et Wieder, A. (2010). Preparing to lead an effective classroom: The role of teacher training and professional development programs. Repéré à http://files.eric.ed.gov/fulltext/ED509718.pdf 
Cave, A. et Mulloy, M. (2010). How do cognitive and motivational factors influence teachers' degree of program implementation? A qualitative examination of teacher perspectives. National Forum of Educational Administration and Supervision Journal, 27(4), 1-26. Repéré à http://www.nationalforum.com/Electronic Journal Volumes/Cave, Agnes A Qualitative Examination of Teacher Perspectives NFEASJ V27 N4 2010.pdf

Charlier, B. (1998). Apprendre et changer sa pratique d'enseignement : expériences d'enseignants. Bruxelles : De Boeck Université.

Deci, E. L. et Ryan, R. M. (2000). The "what" and "why" of goal pursuits: Human needs and the self-determination of behavior. Psychological Inquiry, 11(4), 227-268. Repéré à http://dx.doi.org/10.1207/s15327965pli1104 01

Dionne, L. et Couture, C. (2010). Focus sur le développement professionnel en sciences d'enseignants à l'élémentaire. Apport potentiel de deux communautés d'apprentissage au sentiment d'autoefficacité et au développement professionnel d'enseignants au Canada. Éducation et formation, (293), 151-164. Repéré à http://revueeducationformation.be/include/download.php?idRevue $=9$ \&idRes $=69$

Dionne, L. et Couture, C. (2013). Avantages et défis d'une communauté d'apprentissage pour dynamiser l'enseignement des sciences et de la technologie à l'élémentaire. Éducation et francophonie, 41(2), 212-231. http://dx.doi.org/10.7202/1021034ar

Dionne, L. et Savoie-Zajc, L. (2011). Sens, caractéristiques et retombées de la collaboration entre enseignants et contribution au développement professionnel. Dans L. Portelance, C. Borges et J. Pharand (dir.), La collaboration dans le milieu de l'éducation (p. 45-58). Québec, QC : Presses de l'Université du Québec.

Donnay, J. et Charlier, E. (2008). Apprendre par l'analyse des pratiques : initiation au compagnonnage réflexif (2 $2^{\mathrm{e}}$ éd.). Namur : Presses universitaires de Namur.

Fontaine, S., Savoie-Zajc, L. et Cadieux, A. (2013). L'impact des CAP sur le développement de la compétence des enseignants en évaluation des apprentissages. Éducation et francophonie, 41(2), 10-34. http://dx.doi.org/10.7202/1021025ar

Fullan, M. (2011). The moral imperative realized. Thousand Oaks, CA : Corwin Press.

Karsenti, T. et Larose, F. (2005). L’intégration pédagogique des TIC dans le travail enseignant : recherches et pratiques. Québec, QC : Presses de l'Université du Québec.

Keller, J. M. (1987). Development and use of the ARCS model of instructional design. Journal of Instructional Development, 10(2), 2-10. http://dx.doi.org/10.1007/bf02905780

Leclerc, M. et Labelle, J. (2013). Au cœur de la réussite scolaire : communauté d'apprentissage professionnelle et autres types de communautés. Éducation et francophonie, 41(2), 1-9. http://dx.doi.org/10.7202/1021024ar

Leclerc, M., Philion, R., Dumouchel, C., Laflamme, D. et Giasson, F. (2013). La direction d'école comme pilier à l'implantation de la communauté d'apprentissage professionnelle chez les enseignants en adaptation scolaire au secondaire. Éducation et francophonie, 41(2), 123-154. http://dx.doi.org/10.7202/1021030ar

Ministère de l'Éducation, du Loisir et du Sport du Québec (MELS). (2008). Programme de soutien à la formation continue du personnel scolaire. Québec, QC : Gouvernement du Québec.

Ministère de l'Éducation du Québec (MEQ). (2001). La formation à l'enseignement professionnel, les orientations, les compétences professionnelles. Repéré à http://www.education.gouv.qc.ca/fileadmin/site web/documents/reseau/ formation titularisation/formation enseignement orientations EN.pdf

Moreau, A. C., Leclerc, M. et Stanké, B. (2013). L’apport du fonctionnement en communauté d'apprentissage professionnelle de huit écoles sur l'autoappréciation en enseignement en littératie et sur le sentiment d'autoefficacité. Éducation et francophonie, 41(2), 35-61. http://dx.doi.org/10.7202/1021026ar

Organisation de coopération et de développement économiques (OCDE). (2005). Le rôle crucial des enseignants : attirer, former et retenir des enseignants de qualité. Repéré à http://www.oecd.org/fr/edu/scolaire/ lerolecrucialdesenseignantsattirerformeretretenirdesenseignantsdequalite-rapportfinal.htm

Peters, M. et Savoie-Zajc, L. (2013). Vivre une CAP : appréciations de participants sur les retombées professionnelles perçues. Éducation et francophonie, 41(2), 102-122. http://dx.doi.org/10.7202/1021029ar 
Poupart, J. (1997). L'entretien de type qualitatif : considérations épistémologiques, théoriques et méthodologiques. Dans J. Poupart, J.-P. Deslauriers, L.-H. Groulx, A. Laperrière, R. Mayer et A. P. Pires (dir.), La recherche qualitative : enjeux épistémologiques et méthodologiques (p. 173-209). Montréal, QC : Gaëtan Morin.

Rhéaume, D., Lavoie, L., Cartier, R., Laurin, P., Parent, G., Royer, C. et Toussaint, T. (1999). Le rôle des universités québécoises en matière de formation continue des enseignants. Qu'en pensent les enseignantes et les enseignants des écoles québécoises?. Vie pédagogique, (111), 52-55.

Roy, M. A., Moussaid, S., Gnofame, A. et Renaud, T. (2013, mai). Une classe alternative au $2^{e}$ cycle du secondaire en formation générale des adultes. Communication présentée au Colloque TREAQ-FP, Québec, QC.

Savoie-Zajc, L. (2010). Les dynamiques d'accompagnement dans la mise en place de communautés d'apprentissage de personnels scolaires. Éducation et formation, (293), 9-20. Repéré à http://revueeducationformation.be/include/download.php?idRevue=9\&idRes $=60$

Schieb, L. J. et Karabenick, S. A. (2011). Teacher motivation and professional development: A guide to resources. Repéré à3Rasmy et Karsenti.rtf http://mspmap.org/wp-content/uploads/2011/10/TeachMotivPD Guide.pdf

Spear, G. E. (1988). Beyond the organizing circumstance: A search for the methodology for the study of self-directed learning. Dans H. B. Long (dir.), Self-directed learning: Application and theory (p. 199-221). Athènes : University of Georgia.

Tashakkori, A. et Teddlie, C. (2003). The past and future of mixed methods research: From data triangulation to mixed model designs. Dans A. Tashakkori et C. Teddlie (dir.), Handbook of mixed methods in social and behavioral research (p. 671-702). Thousand Oaks, CA : Sage.

Van der Maren, J.-M. (1996). Méthodes de recherche pour l'éducation (2éd.). Montréal, QC : Presses de l'Université de Montréal.

Wenger, E. (2005). La théorie des communautés de pratique : apprentissage, sens et identité. Québec, QC : Presses de l'Université Laval.

Wenger, E. (2006) Communities of practice: A brief introduction. Repéré à https://web.archive.org/web/20100210074545/http://www.ewenger.com/theory/index.htm

\section{Notes}

1 Dans ce document, l'emploi du masculin pour désigner des personnes n'a d'autres fins que celle d'alléger le texte.

2 Le MEQ est devenu le MELS en 2005.

3 Deux ans après l'implantation de cette communauté d'apprentissage professionnelle, le taux de réussite des jeunes concernés par cette intervention a connu une nette amélioration par rapport aux années précédentes (Roy et al., 2013).

\section{Pour citer cet article}

Rasmy, A., Karsenti, T. (2017). Effet perçu d'une communauté d'apprentissage sur la motivation des enseignants du secondaire dans leur développement professionnel. Formation et profession, 25(3), 36-52.

http://dx.doi.org/10.18162/fp.2017.352 


\section{ANNEXE}

\section{Guide d'entrevue individuelle}

\section{Introduction}

Présentation des objectifs de l'entrevue situés dans le cadre plus large de la recherche.

\section{Les principaux thèmes de l'entrevue}

Nous aimerions que vous nous parliez de cette formation en nous racontant comment se sont déroulés votre expérience de développement professionnel, votre engagement dans les activités de formation et les relations que vous avez établies avec votre animateur, vos collègues. Nous nous intéressons particulièrement aux éléments positifs et négatifs liés à la motivation de poursuivre le développement professionnel en intégration des TIC. Plus spécifiquement, nous cherchons à connaître les différents aspects de votre motivation sous les angles suivants : sentiment de confiance, développement des compétences professionnelles, climat de formation, pertinence des activités de formation, etc.

- Quelles sont les principales raisons de votre participation à la $\mathrm{CA}$ « Groupe Création »?

- Est-ce que les activités de la CA vous ont encouragé d'une manière ou d'une autre dans la poursuite de cette formation? Comment? En quoi cela a-t-il eu un impact sur votre envie de vous impliquer?

- Parlez-moi un peu des relations que vous avez établies avec votre animateur et vos collègues dans cette formation.

- Les collègues et l'animateur manifestent-ils des capacités d'écoute et de rétroaction?

- Les collègues manifestent-ils de l'empathie les uns envers les autres?

- J'aimerais avoir des renseignements sur les effets de tous ces éléments (que vous venez de citer) sur votre envie de participer à cette formation, votre sentiment dêtre capable de développer vos compétences.

\section{Conclusion de l'entrevue}

Auriez-vous quelque chose à rajouter?

Remerciements et fin de l'entretien.

\section{Des repères}

- Durée de l'entrevue : une trentaine de minutes.

- Autorisation d'enregistrement : assurance de la confidentialité de tout ce qui va se dire pendant l'entretien quant à sa motivation et à ce qui l'a influencée.

- Possibilité d'utiliser une structure temporelle pour aider l'interviewé à raconter.

- Vérifier pendant qu'on laisse parler l'enseignant, que tous les aspects sont touchés; approfondir les thèmes abordés spontanément. 